\title{
Clinical Progress Note: Addressing Prognosis in Advanced Dementia
}

\author{
Aziz A Ansari, DO'*, Daniel H Pomerantz, MD, MPH2,3, Nauzley Abedini, MD, MSc ${ }^{4,5}$, \\ Robert L Jayes, MD'6 Brenda Matti-Orozco, MD7, Rachel D Havyer, MD
}

\begin{abstract}
'Division of Hospital Medicine, Loyola University Medical Center, Maywood, Illinois; ${ }^{2}$ Division of General Internal Medicine and Department of Family Medicine (Palliative Care), Albert Einstein College of Medicine, Bronx, New York; ${ }^{3}$ Department of Medicine, Montefiore New Rochelle Hospital, New Rochelle, New York; ${ }^{4}$ Division of Hospital Medicine, University of Michigan, Ann Arbor, Michigan; ${ }^{5}$ National Clinician Scholars Program, University of Michigan, Ann Arbor, Michigan; ${ }^{6}$ Division of Geriatrics and Palliative Medicine, George Washington University Medical Faculty Associates, Washington, DC; ${ }^{7}$ Division of General Internal Medicine \& Palliative Medicine, Morristown Medical Center, Atlantic Health System, Morristown, New Jersey; ${ }^{8}$ Division of Community Internal Medicine and Center for Palliative Medicine, Mayo Clinic, Rochester, Minnesota.
\end{abstract}

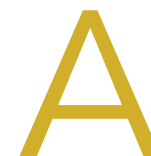

dvanced dementia (AD) is a serious terminal illness. Some features of AD include significant memory deficits (inability to recognize family members), inability to ambulate, very limited verbal communication, and needing assistance with all activities of daily living. ${ }^{1}$ $A D$ carries a 6 -month mortality of $25 \%$ and a median survival of 1.3 years. ${ }^{1}$

Despite a limited life expectancy, patients with AD face increasingly significant symptom burden and use of burdensome interventions ${ }^{2}$ near the end of life. Among the most common interventions hospitalists routinely navigate during a hospitalization is tube feeding for enteral artificial nutrition, which has not been shown to prolong survival, improve quality of life, decrease risk of aspiration pneumonia, or decrease the risk of pressure ulcers. ${ }^{3,4}$ Recent data show that rates of hospitalizations in the last 90 days of life, especially in the last 3 days of life, are increasing. ${ }^{5}$ These late transitions can have significant negative impact on family perceptions of quality of care, including not being treated with respect, receiving care inconsistent with goals, receiving inadequate communication about care decisions, and not being fully informed of the medical conditions. ${ }^{6}$

Therefore, hospitalization in $A D$, especially a readmission, indicates a critical change in a patient's illness, marking an opportune time to have discussions on prognosis and improve care at the end of life. While determining and sharing prognosis can be challenging in the setting of many chronic diseases, resources exist to help clinicians share prognosis in $A D$ and understand the goals of care for each patient.? The aim of this paper is to assist hospitalists in addressing prognosis in the setting of $A D$. We identify and present key knowledge and recommendations from relevant articles identified from a hand-search of articles, published in 2018, from leading palliative care journals, as well as a MEDLINE search from 2003 through December 2018 using the key words "dementia" and

*Corresponding Author: Aziz A Ansari, DO, SFHM, FAAHPM, FACP; Email: Aansar1@lumc.edu; Telephone: 708-216-5118.

Published online first October 23, 2019

Find Additional Supporting Information in the online version of this article.

Received: July 9, 2019; Revised: August 21, 2019; Accepted: August 23, 2019

(๑) 2019 Society of Hospital Medicine DOI 10.12788/jhm.3316 "prognosis." Final presented articles and recommendations were determined based on scientific rigor and relevance to hospital-based care of patients with $A D$.

\section{IMPORTANCE OF PROGNOSIS DISCUSSIONS IN ADVANCED DEMENTIA}

For a myriad of reasons, most $A D$ caregivers do not receive adequate information on the complications of dementia or prognosis. ${ }^{2}$ Conversations that provide prognostic estimates and aim to understand the goals, preferences, and values of $A D$ patients and their surrogates can help in providing goal-concordant care. A prospective study of nursing-home patients with $A D$ showed that having goals of care discussions was strongly associated with surrogates' likelihood of estimating a life expectancy of less than 6 months in AD patients. ${ }^{8} \mathrm{Hav}$ ing this perception was associated with a lower likelihood of patients with $A D$ undergoing burdensome interventions such as hospitalizations, parenteral therapy, venipuncture, feeding tube, or urinary catheterization. ${ }^{8}$ To help improve goal-concordant care, it is important that hospitalists be prepared to have prognostic conversations with patients and their caregivers.

\section{"FORESEEING" PROGNOSIS IN ADVANCED DEMENTIA}

Offering a clinical prognosis involves components of foreseeing (estimating prognosis) and foretelling (sharing prognosis). ${ }^{9}$ Foreseeing prognosis in $\mathrm{AD}$ can be complex due to the highly variable but slow, dwindling clinical course of $A D$. As a practical matter, determining if a patient has a 6-month prognosis is most helpful as eligibility for hospice services may allow for a discharge to a supportive home setting instead of a transfer to an institution. ${ }^{10}$ An evidence-based clinical prediction rule, the Advanced Dementia Prognostic Tool (ADEPT, Appendix Table), ${ }_{1}^{11}$ can be used to estimate prognosis by a composite of 12 risk factors in nursing-home patients. Although the consensus-based National Hospice and Palliative Care Organization (NHPCO) guidelines for Medicare hospice eligibility ${ }^{12}$ (Table) do not perform well in predicting individual mortality, they are used as criteria for hospice enrollment. Given the variability of course of $A D$, evaluating the mortality risk for acute illnesses leading to hospitalization, like pneumonia or hip fracture, can further help estimate prognosis. The website, www.eprognosis. 
TABLE. Foreseeing Prognosis by Staging Dementia and Determining Medicare Hospice Eligibility ${ }^{12}$

FAST tool for dementia
Stages
1 - No difficulties
2 - Subjective forgetfulness
3 - Decreased job functioning and organizational capacity
4 - Difficulty with complex tasks and instrumental ADLs
5 - Required supervision of ADLs
6 - A. Cannot dress without assistance
B. Cannot bathe without assistance
C. Cannot perform toileting without assistance
D. Incontinent of urine
E. Incontinent of bowel
7 - A. Ability to speak limited to six words
B. Ability to speak limited to one word
C. Loss of ambulation
D. Inability to sit
E. Inability to smile
F. Inability to hold head up

Medicare hospice benefit eligibility criteria for dementia

Patients will be considered in the terminal stage of dementia (prognosis of 6 months or less) if they meet the following two criteria regarding the FAST tool and medical conditions:
1. Patients must be at or beyond stage $7 \mathrm{C}$ on the FAST tool and have all the features of stage $6 \mathrm{~A}$ through $7 C$ (see first column):

\section{AND}

2. Patients must have had at least one of the following medical conditions in the past 12 months:

Aspiration pneumonia

Pyelonephritis or another upper urinary tract infection

Septicemia

Decubitus ulcers, multiple, stage 3 or 4

Fever, recurrent after antibiotics; and/ or

Inability to maintain enough fluid and calorie intake with $10 \%$ weight loss during the previous 6 months, or serum albumin $<2.5 \mathrm{~g} / \mathrm{dL}$

Abbreviations: ADLs, activities of daily living; FAST, functional assessment staging.

com, combines various prediction tools to help estimate prognosis. Although using these tools can often help clinicians satisfy the entry requirements to offer hospice, the ADEPT tool and the NHPCO criteria both perform poorly in discriminating those who will or will not actually die in six months. ADEPT, as a prognostic tool, has not yet been validated for community-dwelling patients. Clinicians should exercise caution in making a highly specific estimate of survival in AD, but can and should communicate the expected decline in function over time.

\section{"FORETELLING" PROGNOSIS IN ADVANCED DEMENTIA}

Having goals of care conversations and sharing prognosis has many benefits. A large multistate cohort study showed that having goals of care conversations among patients with terminal cancer was associated with less use of intensive care units, mechanical ventilation, and cardiopulmonary resuscitation. ${ }^{13}$ Caregivers may also benefit from prognosis discussions through identifying resources to care for the patient at home and by potentially limiting their risk of major depressive order and regret, common among those witnessing patients undergoing aggressive treatment at the end of life. ${ }^{13}$ How to share prognosis can be challenging; however, tools such as the Serious IIIness Conversation Guide ${ }^{14}$ can provide step-by-step guidance for providers. The key aspects of the guide are asking permission, assessing illness understanding, and exploring goals, fears, worries, and tradeoffs.

Before exploring goals, it is helpful to explain the serious illness by using "I wish," "I worry," and "I wonder" statements such as "I wish I had better news for you; your mom's dementia has progressed given the recent complication of aspiration," "I worry that she will not be able to eat on her own and will develop another serious infection very soon," or "I wonder whether it is a good time to talk about what your mom would want if she cannot eat on her own."

An example of an effective conversation about artificial nutrition and hydration with a surrogate of a patient with $A D$ with recurrent aspirations may include the following elements: ${ }^{14,15}$

- Obtain the caregiver's and/or patient's perception of illness: "Is it OK if we have a conversation about what may lie ahead with your mother? Is there anyone else that should be present? What is your understanding of your mother's illness?"

- Give relevant data: "Based on her current level of decline with complications and repetitive hospitalizations, I am worried that her life expectancy is likely measured in months rather than years."

- Address emotions: "This must be very hard to hear. I cannot imagine how difficult it must be to see her in the hospital so often."

- Elicit concerns and goals based on understanding key values: "Tell me what you are hoping for regarding your mother's future care and what worries you have. Tell me what your mother would say if she could fully understand her current situation."

- Present goals based on patient and caregiver values: "Based on what you have told me about your mother, she valued her interactions with family and her independence, and she would not want measures that would cause distress, especially when facing a terminal illness."

- Be mindful of prognostic uncertainty: "While we cannot know for certain what will happen next, I am very worried that your mother will continue to aspirate even with a feeding tube."

- Make a recommendation with permission: "From our conversation, I have an idea of what treatment might make sense to your mom. May I share my recommendation with you?" If they are willing, you might say: "As evidence shows 
that feeding tubes do not improve the level of family interaction or independence in patients with dementia and as your mother would not want any distressing procedures, I recommend that we do not place a feeding-tube."

- Balance realism and hope: "Instead, we can focus on other ways to maintain dignity and quality of life for her even without a feeding tube."

\section{RESPONDING TO CHALLENGES}

Conversations about goals of care and prognosis can be challenging and time consuming. At times, the conversations can be strained. The following tips are based on authors' shared experiences to help in those challenging situations:

- Caregivers may show signs of emotional and/or cognitive strain: Recognize and name the emotional response and consider asking the family if they need a break to avoid overtaxing them.

"I can see that this is very difficult for you. Do you want

to take a break and meet again?"

- Caregivers may have unrealistic hopes: Confirm the caregivers' understanding of the situation, before assuming their hope is unrealistic. Try to reframe what they/we can hope for by validating their goals while avoiding unnecessary burdens or discomfort.

"I want to be sure that I have explained your mom's situation clearly. Can you tell me in your words, what I have told you?" as this gives you an opportunity to clarify misunderstandings that may manifest as "false hope".

"Together we can hope for the best and see if your mother can tolerate hand-feeding safely without caus-

ing any harm or distress."

- Avoid assumptions about cultural and religious beliefs: Be curious and demonstrate cultural humility to all patients.

"Are there any cultural or spiritual beliefs that are important to you or your mother?"

- Avoid spending too much time on clinical details: Give families time to share stories about the patient in better days as this gives you an opportunity to get to know the patient.

"Tell me more about what your mother was like when she was healthy."

- Listen first, recommend second: Refrain from making recommendations about the patient's care before you understand his/her values and preferences.

"What would your mother say is most important to her as her health worsens?"

- Use active listening techniques: Using reflection statements can confirm your understanding of the caregiver's view point.

"So, I hear that your mother valued being at home and

being comfortable. Is that correct?"

These conversations are often an iterative process of helping the patient and family traverse the course of AD. Therefore, starting the process even during a hospitalization earlier in the course of $A D$ can help engage in preparedness planning to provide goal-concordant care and help optimize the patient's quality of life.

\section{CONCLUSION}

Hospitalization among patients with AD can signal a significant change in prognosis and represents an important opportunity for further dialogue. A patient- and caregiver-centered conversation, sharing prognosis and learning about values important to the patient and family, has the potential to lead to less burdensome interventions. Doing so can minimize harm, promote quality of life, and reduce unnecessary care transitions near the end of life.

Disclosures: The authors have nothing to disclose.

Funding: Dr Havyer was supported, in part, by the Mayo Clinic Department of Medicine Catalyst for Advancing in Academics grant. Dr Abedini was supported by the National Clinician Scholars Program at the Institute for Healthcare Policy and Innovation, University of Michigan, Ann Arbor, MI.

\section{References}

1. Mitchell SL. Advanced dementia. N Engl J Med. 2015;373(13):1276-1277. https://doi.org/10.1056/NEJMcp1412652

2. Mitchell SL, Teno JM, Kiely DK, et al. The clinical course of advanced dementia. N Engl J Med. 2009;361(16):1529-1538. https://doi.org/10.1056/NEJMoa0902234

3. Teno JM, Gozalo PL, Mitchell SL, et al. Does feeding tube insertion and its timing improve survival? J Am Geriatr Soc. 2012;60(10):1918-1921. https:// doi.org/10.1111/j.1532-5415.2012.04148.x

4. Teno JM, Gozalo P, Mitchell SL, Kuo S, Fulton AT, Mor V. Feeding tubes and the prevention or healing of pressure ulcers. Arch Intern Med. 2012;172(9):697-701. https://doi.org/10.1001/archinternmed.2012.1200

5. Teno JM, Gozalo PL, Bynum JP, et al. Change in end-of-life care for medicare beneficiaries: site of death, place of care, and health care transitions in 2000, 2005, and 2009. JAMA. 2013;309(5):470-477. https://doi.org/10.1001/ jama.2012.207624

6. Makaroun LK, Teno JM, Freedman VA, Kasper JD, Gozalo P, Mor V. Late transitions and bereaved family member perceptions of quality of end-of-life care. $J$ Am Geriatr Soc. 2018;66(9):1730-1736. https://doi.org/10.1111/jgs.15455

7. Ansari AA, Pomerantz DH, Jayes RL, Aguirre EA, Havyer RD. Promoting primary palliative care in severe chronic obstructive pulmonary disease: symptom management and preparedness planning. J Palliat Care. 2019;34(2):8591. https://doi.org/10.1177/0825859718819437

8. Loizeau AJ, Shaffer ML, Habtemariam DA, Hanson LC, Volandes AE, Mitchell SL. Association of prognostic estimates with burdensome interventions in nursing home residents with advanced dementia. JAMA Intern Med. 2018;178(7):922-929. https://doi.org/10.1001/jamainternmed.2018.1413

9. Glare PA, Sinclair CT. Palliative medicine review: Prognostication. J Palliat Med. 2008;11(1):84-103. https://doi.org/10.1089/jpm.2008.9992

10. Jayes RL, Arnold RM, Fromme EK. Does this dementia patient meet the prognosis eligibility requirements for hospice enrollment? J Pain Symptom Manage. 2012;44(5):750-756. https://doi.org/10.1016/j.jpainsymman.2012.08.004

11. Mitchell SL, Miller SC, Teno JM, Kiely DK, Davis RB, Shaffer ML. Prediction of 6-month survival of nursing home residents with advanced dementia using ADEPT vs hospice eligibility guidelines. JAMA. 2010;304(17):1929-1935. https://doi.org/10.1001/jama.2010.1572

12. Schonwetter RS, Han B, Small BJ, et al. Predictors of six-month survival among patients with dementia: an evaluation of hospice Medicare guidelines. Amer J Hospice \& Pall Care. 2003;20(2):105-113. https://doi. org/10.1177/104990910302000208

13. Wright AA, Zhang B, Ray A, et al. Associations between end-of-life discussions, patient mental health, medical care near death, and caregiver bereavement adjustment. JAMA. 2008;300(14):1665-1673. https://doi.org/10.1001/ jama.300.14.1665

14. Bernacki R, Hutchings $M$, Vick J, et al. Development of the serious illness care program: a randomised controlled trial of a palliative care communication intervention. BMJ Open. 2015;5(10):e009032-2015-009032. https://doi. org/10.1136/bmjopen-2015-009032

15. Ansari A, Pomerantz D, Smith K. Being mindful: difficult decisions in advanced dementia and end stage renal disease. SGIM Forum. 2017;40(3):4, 13. 